\title{
EXACT SOLUTIONS FOR REDISTRIBUTION BY NONLINEAR CONVECTION-DIFFUSION
}

\author{
J. R. PHILIP ${ }^{1}$
}

(Received 25 July 1990; revised 11 October 1990)

\begin{abstract}
The nonlinear convection-diffusion equation has been studied for 40 years in the context of nonhysteretic water movement in unsaturated soil. We establish new similarity solutions for instantaneous sources of finite strength redistributed by nonlinear convection-diffusion obeying the dimensionless equation $\partial \theta / \partial t=$ $\partial\left(\theta^{m} \partial \theta / \partial z\right) / \partial z-\theta^{m+1} \partial \theta / \partial z(m \geq 0)$. For $m=0$ (Burgers' equation) solutions involve the error function, and for $m=1$ Airy functions. Problems 1 , 2 , and 3 relate, respectively, to the regions $0 \leq z \leq \infty,-\infty \leq z \leq \infty$, and $-\infty \leq z \leq 0$. Solutions for $m=0$ have infinite tails, but for $m>0$ and finite $t, \theta>0$ inside, and $\theta=0$ outside, a finite interval in $z$. At the slug boundary, $\theta(z)$ is tangential to the $z$-axis for $0<m<1$; and it meets the axis obliquely for $m=1$ and normally for $m>1$. Illustrative results are presented. For Problems 1 and 2 (but not 3 ) finiteness of source strength sets an upper bound on $\boldsymbol{\theta}_{0}$, the similarity "concentration" at $z=0$. The magnitude of convection relative to diffusion increases with $\theta_{0}$; and apparently the dynamic equilibrium between the two processes, implied by the similarity solutions, ceases to be possible when $\theta_{0}$ is large enough.
\end{abstract}

\section{Introduction}

The current interest of mathematicians in nonlinear diffusion and nonlinear convection-diffusion was preceded many decades earlier by that of physicists concerned with soil-water. Building on the pioneering physical concepts of Buckingham [2], Richards [17] in 1931 developed the general partial differential equation describing water flow in unsaturated soils. For nonhysteretic flows in homogeneous isotropic soils this is the nonlinear Fokker-Planck, or

\footnotetext{
${ }^{1}$ CSIRO Centre for Environmental Mechanics, Canberra, ACT 2601, Australia.

(C) Copyright Australian Mathematical Society 1992, Serial-fee code 0334-2700/92
} 
convection-diffusion, equation

$$
\frac{\partial \theta}{\partial t_{*}}=\nabla_{*} \cdot\left(D \nabla_{*} \theta\right)-\frac{d K}{d \theta} \cdot \frac{\partial \theta}{\partial z_{*}}
$$

Here $\theta$ is the volumetric moisture content, $t_{*}$ is time, and $z_{*}$ is the vertical space coordinate, taken positive downward. $\nabla_{*}$ signifies differentiation with respect to space coordinates. (We drop asterisks later when we introduce dimensionless variables.) $D$, the moisture diffusivity, and $K$, the hydraulic conductivity, are strongly-varying functions of $\theta$. There is a very large literature on the solution of (1.1) in soil physics and hydrology, which goes back to the early 1950 s. References [11-16] give extensive reviews.

For a given soil $D(\theta)$ and $K(\theta)$ are experimentally measured functions which characterise it hydrologically. $D$ and $K$ are inherently non-negative, with $d K / d \theta$ a nondecreasing function of $\theta$. The primary emphasis has been on seeking solutions for $D(\theta)$ and $K(\theta)$ otherwise arbitrary. This has been supplemented by work with these functions assigned simple functional forms which, it is hoped, may model adequately actual measured functions for a particular soil. The power-law form of $D(\theta)$ is popular with mathematicians. Its implications in the soil-water context were explored as early as 1957 [10]. Positive power laws yield some simple and interesting results, mainly because they require that $D \rightarrow 0$ as $\theta \rightarrow 0$. For soils, $D$ frequently tends to be small at the dry end of the moisture range, but it is definitely not zero.

Soil physicists have worked more with infiltration problems, with either moisture content or flow velocity prescribed at the soil surface, than with the (generally more difficult) problems of redistribution. The latter deal with the subsequent redistribution of a finite slug of water initially in the soil mass. This slug may have been infiltrated at the surface by rainfall or irrigation, or at some depth beneath the surface by sub-irrigation or in an experimental arrangement, or experimentally at the bottom of a soil column. We designate these as Problem 1 (downward redistribution), Problem 2 (two-way redistribution), and Problem 3 (upward redistribution).

We develop here solutions bearing on these three distinct modes of redistribution. Because they are similarity solutions, we necessarily follow the mathematicians' path by using power-law representations of $D$ and $d K / d \theta$, and we are obliged also to avoid the complication of hysteresis. Accordingly, the results do not apply to coarse-textured soils where capillary hysteresis (e.g. [4]) is important. On the other hand, they are relevant to redistribution in nonhysteretic fine-textured soils, and to the full range of other nonhysteretic nonlinear convection-diffusion processes.

In physical applications, we are generally concerned with the redistribution of an initial slug of the transported material with finite maximum 
concentration and (usually) finite dimensions. The basic solutions generated here are for nonlinear convection-diffusion from an instantaneous point source at $t_{*}=0$. It will be understood that finite slugs initially at appropriate values of $t_{*}>0$ may be matched, as required, to these solutions.

In what follows, we broaden our definitions to apply to any convectiondiffusion process. $D$ is simply a diffusivity, $K$ a convective velocity, $\theta$ the volume fraction of the transported material (based, if necessary, on a putative material density), and $z_{*}$ the space coordinate taken positive in the direction of the convective velocity. We continue to consider the three distinct Problems 1, 2, and 3; and we retain the requirements that $\theta, D$, and $K$ be non-negative and that $d K / d \theta$ be a nondecreasing function of $\theta$.

\section{Similarity solutions for redistribution by convection-diffusion}

We use the one-dimensional $\left(z_{*}\right)$ form of $(1.1)$ with

$$
D=D_{0} \theta^{m}, \quad \frac{d K}{d \theta}=k \theta^{n}, \quad\left(D_{0}>0, k>0, m \geq 0, n \geq 0\right)
$$

namely

$$
\frac{\partial \theta}{\partial t_{*}}=\frac{\partial}{\partial z_{*}}\left(D_{0} \theta^{m} \frac{\partial \theta}{\partial z_{*}}\right)-k \theta^{n} \frac{\partial \theta}{\partial z_{*}} .
$$

We reduce this through the substitutions

$$
z=k z_{*} / D_{0}, \quad t=k^{2} t_{*} / D_{0},
$$

to the dimensionless form

$$
\frac{\partial \theta}{\partial t}=\frac{\partial}{\partial z}\left(\theta^{m} \frac{\partial \theta}{\partial z}\right)-\theta^{n} \frac{\partial \theta}{\partial z}
$$

This is subject to the initial condition

$$
t=0, z \neq 0, \theta=0 \text {. }
$$

We seek similarity solutions of (2.4), (2.5) describing redistribution of a finite quantity of the transported material. The appropriate form is

$$
\theta=\Theta(\varphi) t^{-\alpha}, \quad \varphi=z t^{-\alpha}, \quad(\alpha>0)
$$

which ensures total material conservation. Putting (2.6) into (2.4), we find that similarity requires that

$$
1+\alpha=(m+3) \alpha=(n+2) \alpha .
$$

That is, we have

$$
n=m+1, \quad \alpha=1 /(m+2) .
$$


(Note that taking $n=m+1$ is, in the soil-water context, equivalent to taking $d \Psi / d \theta$, the derivative of the moisture potential $\Psi$, proportional to $\theta^{-2}$, a reasonably plausible representation.) We therefore rewrite $(2.4),(2.6)$ as

$$
\begin{aligned}
& \frac{\partial \theta}{\partial t}=\frac{\partial}{\partial z}\left(\theta^{m} \frac{\partial \theta}{\partial z}\right)-\theta^{m+1} \frac{\partial \theta}{\partial z} \\
& \theta=\theta(\varphi) t^{-1 /(m+2)}, \quad \varphi=z t^{-1 /(m+2)} .
\end{aligned}
$$

We see, further, that (2.6) (and (2.10)) enables total material conservation in each of the separate regions $z<0$ and $z>0$. Accordingly, the solutions of (2.9) we seek satisfy the condition of zero flow across $z=0$, namely

$$
t>0, \quad z=0, \quad \frac{\partial \theta}{\partial z}=\frac{\theta^{2}}{m+2} .
$$

Substituting (2.10) into (2.9) and (2.11), we obtain the ordinary differential equation

subject to

$$
\frac{d}{d \varphi}\left[\Theta^{m} \frac{d \Theta}{d \varphi}\right]=\frac{1}{m+2} \frac{d}{d \varphi}\left[\Theta^{m+2}-\varphi \Theta\right]
$$

$$
\varphi=0, \quad \frac{d \Theta}{d \varphi}=\frac{\Theta^{2}}{m+2} .
$$

Integration of (2.12) with respect to $\varphi$, and use of (2.13), then gives

$$
\frac{d \Theta}{d \varphi}=\frac{1}{m+2}\left[\Theta^{2}-\Theta^{1-m} \varphi\right] \text {. }
$$

We seek solutions of (2.14) subject to the condition

$$
\varphi=0, \quad \Theta=\theta_{0}>0 .
$$

$\Theta_{0}$ serves to parametrise the solution. For $m>0,(2.14),(2.15)$ may be rewritten, with $u$ for $\Theta^{m}$, as

$$
\frac{d u}{d \varphi}=\frac{m}{m+2}\left[u^{(m+1) / m}-\varphi\right]
$$

subject to

$$
\varphi=0, \quad u=\Theta_{0}^{m}=u_{0}>0 .
$$

For Problem 1, we require the solution in $0 \leq \varphi \leq \infty$; for Problem 2 in $-\infty \leq \varphi \leq \infty$; and for Problem 3 in $-\infty \leq \varphi \leq 0$. The practical interest is in solutions with $0 \leq \theta<\infty$, and with the integrals

$$
Q_{+}=\int_{0}^{\varphi_{+}} \Theta d \varphi, \quad Q_{-}=\int_{\varphi_{-}}^{0} \boldsymbol{\theta} d \varphi
$$

finite. In connection with Problem 2, we use the notation

$$
Q=Q_{+}+Q_{-} \text {. }
$$


Here $\varphi_{+}$is either the smallest positive finite value of $\varphi$ at which $\theta=0$, or, if this does not exist, $+\infty$; and $\varphi_{-}$is either the negative finite value of $\varphi$ of smallest magnitude at which $\theta=0$, or, if this does not exist, $-\infty$. Note that $Q_{+}, Q$, and $Q_{-}$are respectively the dimensionless source strengths (or slug magnitudes) for Problems 1, 2, and 3. They are related to the physical source strengths (or slug magnitudes) $Q_{*+}, Q_{*}$, and $Q_{*-}$ by the relations

$$
\frac{Q_{*}}{Q}=\frac{Q_{*+}}{Q_{+}}=\frac{Q_{*-}}{Q_{-}}=\frac{D_{0}}{k} .
$$

The $Q_{*}$ 's have the dimensions [length], consistent with $\theta$ expressed as volume fraction.

Beyond our concern with practically-relevant solutions, we need also to examine those solutions for which $\theta \rightarrow \infty$ as $\varphi \rightarrow+\infty$. As we shall see, these singular solutions determine the upper bound on the values of $\theta_{0}$ permissible in Problems 1 and 2.

In the following section, we examine the cases $m=0$ and $m=1$, which provide a useful entrée into the general question of solutions for arbitrary $m>0$.

\section{The special cases $m=0$ and $m=1$}

3.1 The case $m=0$

For $m=0,(2.14)$ reduces to the Bernoulli equation

$$
\frac{d \Theta}{d \varphi}=\frac{1}{2}\left[\Theta^{2}-\Theta \varphi\right]
$$

The solution satisfying $(2.15)$ is

$$
\Theta=\frac{\exp \left(-\frac{1}{4} \varphi^{2}\right)}{\Theta_{0}^{-1}-\frac{\sqrt{ } \pi}{2} \operatorname{erf} \frac{\varphi}{2}}
$$

3.1.1 The upper bound on $\Theta_{0}$. For $\Theta$ to be finite everywhere in $0 \leq \varphi \leq$ $\infty$, we require that

$$
\Theta_{0}^{-1}>\sqrt{ } \pi / 2 \text {, i.e. } 0<\theta_{0}<\theta_{0}^{*}=2 / \sqrt{ } \pi \text {. }
$$

There is thus a definite upper bound on $\boldsymbol{\theta}_{0}, \boldsymbol{\theta}_{0}^{*}$, for the existence of relevant solutions to Problems 1 and 2; but relevant solutions to Problem 3 exist for all $0<\theta_{0}<\infty$. 
3.1.2 Source strengths. Putting (3.2) into (2.18) and (2.19), we find

$$
\begin{aligned}
& Q_{+}=-\ln \left(1-\frac{\sqrt{ } \pi}{2} \Theta_{0}\right), \\
& Q=\ln \left[\left(1+\frac{\sqrt{ } \pi}{2} \theta_{0}\right) /\left(1-\frac{\sqrt{ } \pi}{2} \Theta_{0}\right)\right], \\
& Q_{-}=\ln \left(1+\frac{\sqrt{ } \pi}{2} \theta_{0}\right) .
\end{aligned}
$$

3.1.3 Value and location of $\theta_{\max }$. For $0<\theta_{0}<2 / \sqrt{ } \pi$, $\theta$ has a maximum $\theta_{\max }$ in $0<\varphi<\infty$ [i.e. for Problems 1 and 2]. In view of (3.1), this maximum is at $\varphi=\varphi_{\dagger}=\theta_{\max }$. We see from (3.1) and (3.2) that $\varphi_{\dagger}$ is the root of the transcendental equation

$$
\varphi^{-1} \exp \left(-\frac{1}{4} \varphi^{2}\right)+\frac{\sqrt{ } \pi}{2} \operatorname{erf}\left(\frac{1}{2} \varphi\right)=\Theta_{0}^{-1} .
$$

For small $\boldsymbol{\theta}_{0}$ this reduces to

$$
\Theta_{\max }=\varphi_{\dagger}=\theta_{0}+\frac{1}{4} \Theta_{0}^{3}+\frac{11}{96} \Theta_{0}^{5}+O\left(\Theta_{0}^{7}\right) .
$$

As $\boldsymbol{\theta}_{0} \rightarrow 2 / \sqrt{ } \pi, \boldsymbol{\theta}_{\max }$ and $\varphi_{\dagger}$ increase indefinitely but very slowly. Putting asymptotic expansion [5]

$$
\operatorname{erf}\left(\frac{1}{2} \varphi\right) \sim 1-\frac{2}{\sqrt{ } \pi} \exp \left(-\frac{1}{4} \varphi^{2}\right)\left(\frac{1}{\varphi}-\frac{2}{\varphi^{3}}+\ldots\right)
$$

in (3.7) gives, in this limit,

$$
\varphi_{\dagger}^{3} \exp \left(\frac{1}{4} \varphi_{\dagger}^{2}\right) \sim \frac{4}{\sqrt{ } \pi} /\left(1-\frac{\sqrt{ } \pi}{2} \Theta_{0}\right)
$$

Note that Problem 3, for all $0<\theta_{0}<\infty$, gives $\theta$ increasing monotonically from zero as $\varphi \rightarrow-\infty$ to $\theta_{0}$ at $\varphi=0$. There is no maximum.

3.1.4 Some general properties. For $m=0$, all solutions giving finite $Q$ 's have infinite tails. We note that

$$
\lim _{\varphi \rightarrow-\infty} \exp \left(\frac{1}{4} \varphi^{2}\right)\left(\Theta_{0}^{-1}+\frac{\sqrt{ } \pi}{2}\right) \Theta=1
$$

for all $0<\theta_{0}<\infty$; and that

$$
\lim _{\varphi \rightarrow \infty} \exp \left(\frac{1}{4} \varphi^{2}\right)\left(\theta_{0}^{-1}-\frac{\sqrt{ } \pi}{2}\right) \theta=1
$$

for all $0<\theta_{0}<\sqrt{ } \pi / 2$.

For $m=0, \alpha=1 / 2$, so that $\theta$ at fixed $\varphi$ decreases as $t^{-1 / 2}$ and $z$ at fixed $\theta$ increases as $t^{1 / 2}$.

Note that for $m=0,(2.9)$ reduces to Burgers' equation [3]. The relevance of Burgers' equation to soil-water problems has been recognised for some decades $[13,14]$; but the present redistribution solutions appear to be new. 
3.1.5 The singular case $\boldsymbol{\theta}_{0}=\Theta_{0}^{*}=2 / \sqrt{ } \pi$. The solution for this case is

$$
\Theta=\frac{2}{\sqrt{ } \pi} \frac{\exp \left(-\frac{1}{4} \varphi^{2}\right)}{\operatorname{erfc}\left(\frac{1}{2} \varphi\right)}
$$

with $\theta$ increasing monotonically from 0 at $\varphi=-\infty$ to $\infty$ at $\varphi=+\infty$. The relevant asymptotic expansions, which follow from (3.9), are: $\varphi$ large and negative,

$$
\Theta \sim\left[\sqrt{ } \pi \exp \left(\frac{1}{4} \varphi^{2}\right)+\frac{1}{\varphi}-\frac{2}{\varphi^{3}}+\frac{12}{\varphi^{5}}-\ldots\right]^{-1}
$$

$\varphi$ large and positive,

$$
\Theta \sim \varphi+\frac{2}{\varphi}+\frac{8}{\varphi^{3}}+\ldots
$$

3.2 The case $m=1$

For $m=1,(2.13)$ reduces to the Riccati equation

$$
d \Theta / d \varphi=\left[\Theta^{2}-\varphi\right] / 3
$$

The solution satisfying $(2.15)$ is

$$
\Theta=-3^{1 / 3} \frac{\sqrt{ } 3\left(p+\Theta_{0}\right) A i^{\prime}\left(3^{-2 / 3} \varphi\right)+\left(p-\Theta_{0}\right) B i^{\prime}\left(3^{-2 / 3} \varphi\right)}{\sqrt{ } 3\left(p+\Theta_{0}\right) A i\left(3^{-2 / 3} \varphi\right)+\left(p-\Theta_{0}\right) B i\left(3^{-2 / 3} \varphi\right)}
$$

Here $A i, B i$, are the Airy functions (e.g. [1]), $A i^{\prime}, B i^{\prime}$, their derivatives, and

$$
p=3^{2 / 3} \Gamma\left(\frac{2}{3}\right) / \Gamma\left(\frac{1}{3}\right) \approx 1.051416 .
$$

3.2.1 The upper bound on $\boldsymbol{\theta}_{0}$. When $\boldsymbol{\theta}_{0}>p$, the denominator of the right of (3.17) is zero for some large enough, finite value of $\varphi$. It follows that, for $\Theta$ to be finite everywhere in $0 \leq \varphi \leq \infty$, we require that

$$
0<\theta_{0}<\theta_{0}^{*}=p \approx 1.051416
$$

Here also there is a definite upper bound on $\boldsymbol{\theta}_{0}, \boldsymbol{\theta}_{0}^{*}$, for the existence of relevant solutions to Problems 1 and 2; and relevant solutions to Problem 3 exist for all $0<\theta_{0}<\infty$. 
3.2.2 Source strengths. Putting (3.17) into (2.18) and (2.19) we find

$$
\begin{aligned}
& Q_{+}=-3 \ln \left[\frac{\Gamma\left(\frac{1}{3}\right)}{2 \sqrt{ } 3}\left\{\sqrt{ } 3\left(p+\Theta_{0}\right) A i\left(3^{-2 / 3} \varphi_{+}\right)+\left(p-\Theta_{0}\right) B i\left(3^{-2 / 3} \varphi_{+}\right)\right\}\right], \\
& Q=3 \ln \left[\frac{\sqrt{ } 3\left(p+\Theta_{0}\right) A i\left(3^{-2 / 3} \varphi_{-}\right)+\left(p-\Theta_{0}\right) B i\left(3^{-2 / 3} \varphi_{-}\right)}{\sqrt{ } 3\left(p+\Theta_{0}\right) A i\left(3^{-2 / 3} \varphi_{+}\right)+\left(p-\Theta_{0}\right) B i\left(3^{-2 / 3} \varphi_{+}\right)}\right], \\
& Q_{-}=3 \ln \left[\frac{\Gamma\left(\frac{1}{3}\right)}{2 \sqrt{ } 3}\left\{\sqrt{ } 3\left(p+\Theta_{0}\right) A i\left(3^{-2 / 3} \varphi_{-}\right)+\left(p-\Theta_{0}\right) B i\left(3^{-2 / 3} \varphi_{-}\right)\right\}\right]_{(3 .}
\end{aligned}
$$

Here $\varphi_{+}, \varphi_{-}$are, respectively, the positive root and the negative root of smallest magnitude of

$$
\frac{B i^{\prime}\left(3^{-2 / 3} \varphi\right)}{A i^{\prime}\left(3^{-2 / 3} \varphi\right)}=-\frac{\sqrt{ } 3\left(p+\Theta_{0}\right)}{p-\Theta_{0}} .
$$

3.2.3 Value and location of $\boldsymbol{\Theta}_{\max }$. For $0<\boldsymbol{\theta}_{0}<p, \boldsymbol{\theta}$ has a maximum $\Theta_{\max }$ in $0 \leq \varphi \leq \infty$ [i.e. for Problems 1 and 2]. In view of (3.16), this maximum is at $\varphi=\varphi \dagger=\theta_{\max }^{2}$. Putting $\Theta=\varphi^{1 / 2}$ in (3.17) gives the transcendental equation for $\varphi \dagger$. Problem 3, for all $0<\theta_{0}<\infty$, gives $\theta$ increasing monotonically from zero at $\varphi=\varphi_{-}$to $\theta_{0}$ at $\varphi=0$, with no maximum.

3.2.4 Some general properties. For $m=1$, all solutions giving finite $Q$ 's have $\varphi_{+}$and $\varphi_{-}$finite. Expansions of $A i^{\prime}$ and $B i^{\prime}$ about the origin indicate that, for small $\bar{\Theta}_{0}$,

$$
\varphi_{-} \approx-\left(6 \Theta_{0}\right)^{1 / 2}+O\left(\Theta_{0}^{2}\right)
$$

and

$$
\varphi_{+} \approx\left(6 \Theta_{0}\right)^{1 / 2}+O\left(\Theta_{0}^{2}\right) .
$$

The asymptotic expansions of $A i^{\prime}$ and $B i^{\prime}$ for large argument [1] show that, as $\theta_{0} \rightarrow p$,

$$
\varphi_{+} \sim\left(\frac{9}{4} \ln \frac{p+\theta_{0}}{p-\Theta_{0}}\right)^{2 / 3} .
$$

We note that it follows from (3.16) that, at $\varphi=\varphi_{-}$and $\varphi_{+}, d \Theta / d \varphi$ assumes the finite values $-\frac{1}{3} \varphi_{-}$and $-\frac{1}{3} \varphi_{+}$. That is, the $\Theta(\varphi)$ curve meets the $\varphi$ axis at a finite angle. At $\varphi=\varphi_{-}$the slope angle is less than $\pi / 2$, and at $\varphi=\varphi_{+}$it is greater than $\pi / 2$.

For $m=1, \alpha=1 / 3$, so that $\theta$ at fixed $\varphi$ decreases as $t^{-1 / 3}$ and $z$ at fixed $\Theta$ increases as $t^{1 / 3}$. 
3.2.5 The singular case $\Theta_{0}=\Theta_{0}^{*}=p$. The solution for this case is

$$
\Theta=-3^{1 / 3} \frac{A i^{\prime}\left(3^{-2 / 3} \varphi\right)}{A i\left(3^{-2 / 3} \varphi\right)}
$$

with $\theta$ increasing monotonically from 0 at $\varphi=\varphi_{-}$to $\infty$ at $\varphi=+\infty$. In this case, $\varphi_{-}$is the negative root of

$$
A i^{\prime}\left(3^{-2 / 3} \varphi\right)=0
$$

of smallest magnitude. It has the value $\varphi_{-} \approx-2.118$. The asymptotic expansions of $A i$ and $A i^{\prime}$ for large argument [1] lead to the expansion for large $\varphi$

$$
\theta \sim \varphi^{1 / 2}+\frac{3}{4 \varphi}+\ldots
$$

We see that $\theta \rightarrow \infty$ as $\varphi \rightarrow \infty$ like $\varphi^{1 / 2}$.

\section{General properties of solutions for $m \geq 0$}

We now explore properties of the solutions for arbitrary values of $m>0$. Relevant solutions to Problem 3 exist for all $0<\theta_{0}<\infty$. On the other hand, Problems 1 and 2 require a finite upper bound on $\theta_{0}, \theta_{0}^{*}$, not only for $m=0$ and 1 , but for all $m \geq 0$. In this connection we develop the following asymptotic analysis.

\subsection{Asymptotic analysis of the singular solution}

We seek the solution of (2.14) which tends to infinity as $\varphi \rightarrow+\infty$, that is, the singular solution with $\Theta_{0}=\Theta_{0}^{*}$. We assume that this solution has an asymptotic expansion for large $\varphi$ in descending powers of $\varphi$ of the form

$$
\Theta \sim a_{1} \varphi^{b_{1}}+a_{2} \varphi^{b_{2}}+\ldots
$$

It follows from the structure of (2.14) that the leading terms of the expansions for $\Theta^{2}$ and for $\Theta^{1-m} \varphi$ must be equal; and that the leading term of the expansion for $d \Theta / d \varphi$ must equal the second term of the expansion for $(m+2)^{-1}\left[\boldsymbol{\Theta}^{2}-\boldsymbol{\theta}^{1-m} \varphi\right]$. Equating the various indices and coefficients, we find

$$
a_{1}=1, \quad a_{2}=\frac{m+2}{(m+1)^{2}}, \quad b_{1}=\frac{1}{m+1}, \quad b_{2}=-1 .
$$

This procedure of equating indices and coefficients may be continued indefinitely to establish the higher terms of expansion (4.1). We limit ourselves 
here to the two leading terms:

$$
\Theta \sim \varphi^{1 /(m+1)}+\frac{m+2}{(m+1)^{2}} \varphi^{-1}+\ldots
$$

Note that (3.15) is exactly (4.3) for $m=0$, and (3.29) is exactly (4.3) for $m=1$, as they should be.

\subsection{Behaviour of $\theta_{0}^{*}(m)$}

The analytical determinations of $\Theta_{0}^{*}(0)$ and $\theta_{0}^{*}(1)$ (Section 3) have been supplemented by numerical calculations for other $m$-values, and also by upper and lower bounds for $\Theta_{0}^{*}(m)$ established by analysis.

4.2.1 The upper bound on $\Theta_{0}^{*}(m)$. We seek the singular solution of (2.16), (2.17) which gives $u \rightarrow \infty$ as $\varphi \rightarrow \infty$. For such a solution, $u$ increases monotonically from $u_{0}$ at $\varphi=0$, to $\infty$ as $\varphi \rightarrow \infty$. So, for $\varphi>0, m>0$,

$$
\frac{d u}{d \varphi}>\frac{m}{m+2}\left[u_{0}^{1 / m} u-\varphi\right]
$$

Now the solution of the linear equation

$$
\frac{d u_{1}}{d \varphi}=\frac{m}{m+2}\left[u_{0}^{1 / m} u_{1}-\varphi\right]
$$

subject to

$$
\varphi=0, u_{1}=u_{0}
$$

is

$$
u_{1}=\left(u_{0}-\frac{m+2}{m} u_{0}^{-2 / m}\right) \exp \left(\frac{m u_{0}^{1 / m} \varphi}{m+2}\right)+u_{0}^{-1 / m} \varphi+\frac{m+2}{m} u_{0}^{-2 / m} .
$$

So $u_{1} \rightarrow \infty$ as $\varphi \rightarrow \infty$ provided

$$
u_{0} \geq\left[\frac{m+2}{m}\right]^{\frac{m}{m+2}} \text {. }
$$

Now if $u_{1} \rightarrow \infty$ for a given $u_{0}$, then $u \rightarrow \infty$ for a smaller $u_{0}$. It therefore follows that

$$
\Theta_{0}^{*}<\left[\frac{m+2}{m}\right]^{1 /(m+2)} .
$$

4.2.2 The lower bound on $\theta_{0}^{*}(m)$. Also, for $\varphi>0, m>1$,

$$
\frac{d u}{d \varphi}<\frac{m}{m+2}\left[u_{0}^{(1-m) / m} u^{2}-\varphi\right]
$$


Now the solution of the Riccati equation

$$
\frac{d u_{1}}{d \varphi}=\frac{m}{m+2}\left[u_{0}^{(1-m) / m} u_{1}^{2}-\varphi\right]
$$

subject to (4.6) is in terms of Airy functions, similar in form to (3.17). Then a comparison argument, similar but converse to the preceding one, shows that, for $m>1$,

$$
\Theta_{0}^{*}>\left[\frac{p_{1}(m+2)}{m}\right]^{1 /(m+2)},
$$

where $p_{1}=3^{1 / 3} \Gamma(2 / 3) / \Gamma(1 / 3) \approx 0.3874382$.

4.2.3 Comparing bounds and calculated values. We thus have the inequalities

$$
\left[\frac{p_{1}(m+2)}{m}\right]^{1 /(m+2)}<\Theta_{0}^{*}<\left[\frac{m+2}{m}\right]^{1 /(m+2)},
$$

with the upper bound valid for $m>0$ and the lower for $m>1$. These bounds are rather weak, though the upper bound could be useful for large $m$. Both bounds approach 1 as $m \rightarrow \infty$, establishing that

$$
\lim _{m \rightarrow \infty} \Theta_{0}^{*}=1 \text {. }
$$

This is consistent with Figure 1, which shows the various calculated results. It is seen that $\Theta_{0}^{*}$ decreases, presumably monotonically, from $2 / \sqrt{ } \pi$ at $m=0$ to 1 as $m \rightarrow \infty$. The small variation of $\theta_{0}^{*}$ as $m$ ranges from 0 to $\infty$ is of some interest.

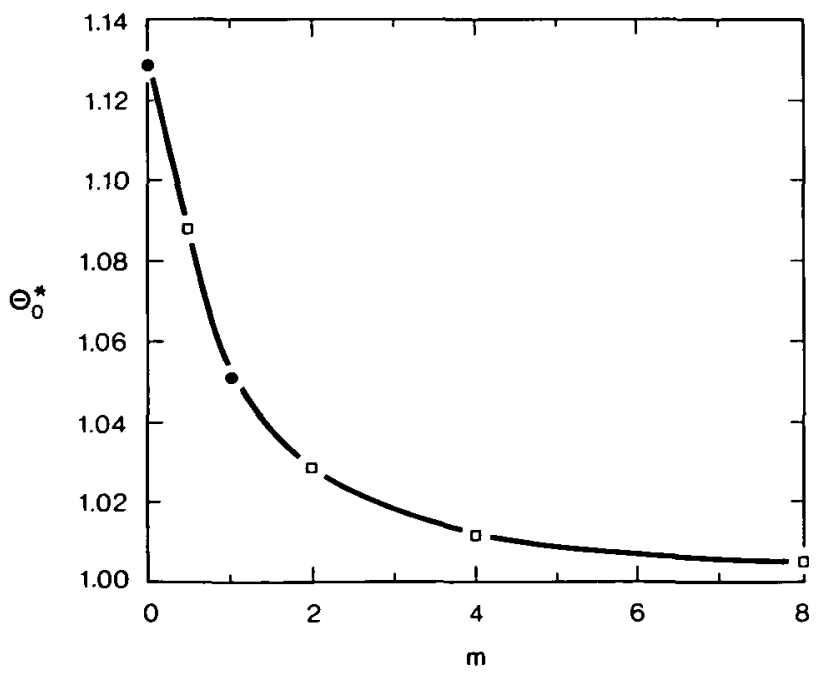

Figure 1. Dependence on $m$ of the upper bound on $\boldsymbol{\theta}_{0}, \boldsymbol{\theta}_{0}^{*}$. Dots: analytical results. Open squares: numerical results. 
4.3 Finiteness of $\varphi_{-}$and $\varphi_{+}$for $m>0$

It is convenient to study the question of the finiteness, or otherwise, of $\varphi_{-}$ and $\varphi_{+}$by reference to (2.16), a form of (2.14) applicable for $m>0$.

Let us suppose that, for some $m>0, u \rightarrow 0$ as $\varphi \rightarrow-\infty$ and/or as $\varphi \rightarrow+\infty$. Then, necessarily, $d u / d \varphi \rightarrow 0$ in the same limit or limits. But (2.16) indicates that, in those circumstances, $d u / d \varphi \rightarrow \pm \infty$ according as $\varphi \rightarrow \mp \infty$. We reach a contradiction. Accordingly $\varphi_{-}$and $\varphi_{+}$must be finite for $m>0$. They are infinite only for $m=0$ (Section 3.1.4).

4.4 Approach of $\theta$ to 0 at $\varphi=\varphi_{-}$and $\varphi_{+}$

We then have from (2.16) that, for $m>0$

$$
\begin{array}{llll}
\varphi=\varphi_{-}, & & u=0, & \frac{d u}{d \varphi}=-\frac{m}{m+2} \varphi_{-} ; \\
\varphi=\varphi_{+}, & u=0, & \frac{d u}{d \varphi}=-\frac{m}{m+2} \varphi_{+} .
\end{array}
$$

Now, since $-\infty<\varphi_{-}<0$ and $0<\varphi_{+}<\infty$,

$$
\begin{aligned}
& \varphi=\varphi_{-}, \quad 0<d u / d \varphi<\infty \\
& \varphi=\varphi_{+}, \quad-\infty<d u / d \varphi<0 \text {. }
\end{aligned}
$$

But

$$
\frac{d \Theta}{d \varphi}=\frac{\Theta^{1-m}}{m} \frac{d u}{d \varphi}
$$

and $\Theta=0$ at $\varphi=\varphi_{-}$and $\varphi_{+}$, so that (4.16) gives

$$
\begin{aligned}
& 0<m<1, \quad \varphi=\varphi_{-} \text {and } \varphi_{+}, \quad d \Theta / d \varphi=0 ; \\
& m=1, \quad \varphi=\varphi_{-}, \quad 0<\frac{d \Theta}{d \varphi}=-\frac{1}{3} \varphi_{-}<\infty, \\
& \varphi=\varphi_{+}, \quad-\infty<\frac{d \Theta}{d \varphi}=-\frac{1}{3} \varphi_{+}<0 ; \\
& m>1, \quad \varphi=\varphi_{-}, \quad d \theta / d \varphi=+\infty, \\
& \varphi=\varphi_{+}, \quad d \Theta / d \varphi=-\infty .
\end{aligned}
$$

We see that, for $0<m<1$, the $\Theta(\varphi)$ curve meets the $\varphi$ axis tangentially at $\varphi=\varphi_{-}$and $\varphi_{+}$; for $m=1$ it meets the axis obliquely (section 3.2.4); and for $m>1$ it meets the axis normally. Figure 2 (see p. 375) compares the solutions with $\Theta_{0}=1$ for $m=0$ (infinite tails), $\frac{1}{2}$ (tangential to axis), 1 (oblique to axis) and 2 (normal to axis).

\subsection{Location of $\boldsymbol{\theta}_{\max }$}

For $0<\theta_{0}<\boldsymbol{\theta}_{0}^{*}, \boldsymbol{\theta}$ has a maximum in $0<\varphi \leq \varphi_{+}$[i.e. for Problems 1 and 2]. In view of (2.14), this maximum is at $\varphi=\varphi_{\dagger}=\Theta_{\max }^{1+m}$. For Problem 


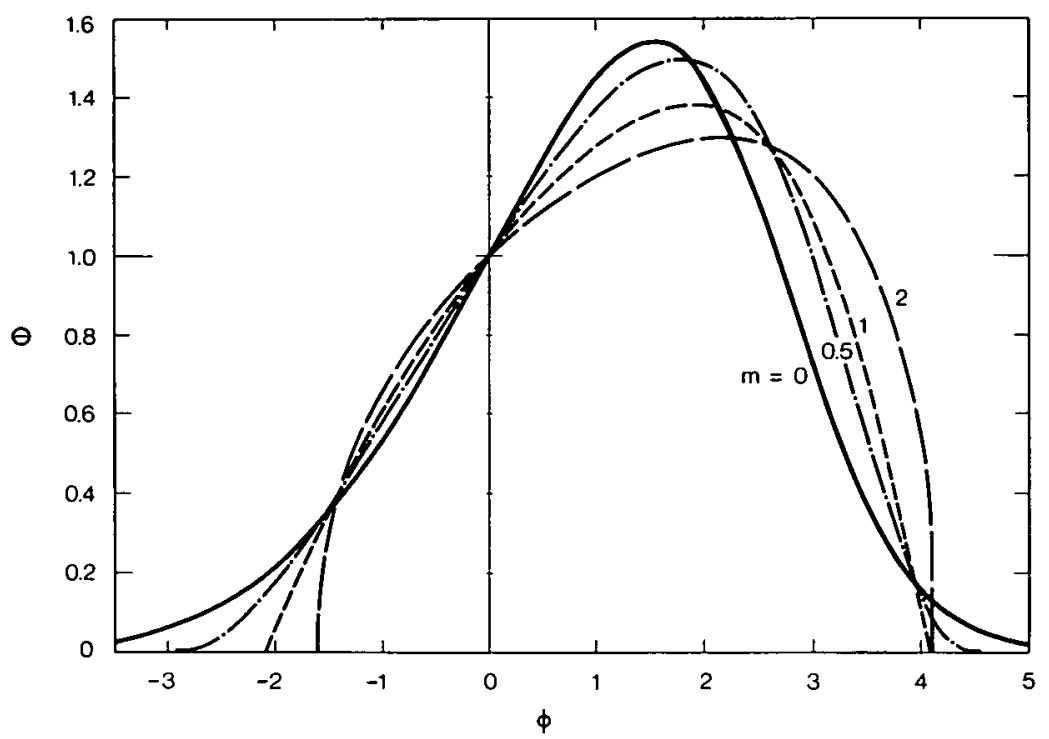

FIgURE 2. Solutions for $\boldsymbol{\theta}_{0}=1$ and $m=0, \frac{1}{2}, 1$, and 2, illustrating the four different modes of approach of $\boldsymbol{\theta}$ to the $\varphi$-axis, according as $m=0,0<m<1, m=1$, or $m>1$.

3, $\Theta$ increases monotonically from 0 at $\varphi=\varphi_{-}$to $\Theta_{0}$ at $\varphi=0$ for all $0<\Theta_{0}<\infty$.

4.6 Expanding the solutions about $\varphi=0$

Repeated differentiation gives Taylor series expansions of the solutions about $\varphi=0$. It is convenient to work with the equation

$$
m>0, \quad d v / d \chi=v^{(m+1) / m}-\chi,
$$

which is (2.14) after the substitutions

$$
v=\left(\frac{m}{m+2}\right)^{m /(m+2)} \Theta^{m}, \quad \chi=\left(\frac{m}{m+2}\right)^{(m+1) /(m+2)} \varphi
$$

Equation (4.19) is subject to the conditions

$$
\chi=0, \quad v=v_{0}=\left(\frac{m+2}{m}\right)^{m /(m+2)} \Theta_{0}^{m}
$$


The leading derivatives of $v$ with respect to $\chi$ at the origin are

$$
\begin{aligned}
v^{\prime}(0) & =v_{0}^{(m+1) / m}, \\
v^{\prime \prime}(0) & =\frac{m+1}{m} v_{0}^{(m+2) / m}-1, \\
v^{\prime \prime \prime}(0) & =v_{0}^{1 / m}\left[\frac{(m+1)(m+2)}{m^{2}} v_{0}^{(m+2) / m}-\frac{m+1}{m}\right], \\
v^{\prime \prime \prime \prime}(0) & =v_{0}^{2 / m}\left[\frac{(m+1)(m+2)(m+3)}{m^{3}} v_{0}^{(m+2) / m}-\frac{(m+1)(m+4)}{m^{2}}\right] .
\end{aligned}
$$

From the Taylor series for $v(\chi)$ which follows, we may derive expansions for $\theta(\varphi)$ of the form

$$
\Theta=\left[\Theta_{0}^{m}+\sum_{1}^{\infty} a_{n}\left(\Theta_{0}, m\right) \varphi^{n}\right]^{1 / m}
$$

These may be usefully convergent, provided $\theta_{0}$ is significantly less than $\theta_{0}^{*}$. The related Taylor series for $v^{\prime}(\chi)$ may, similarly, be used to estimate $\varphi_{\dagger}$ and $\boldsymbol{\theta}_{\max }$.

\subsection{Solution in limit of $\theta_{0}$ small}

For $\theta_{0}$ very small, $\theta$ is very small everywhere in $\varphi_{-} \leq \varphi \leq \varphi_{+}$. In physical terms, this implies that convective transport is negligible relative to diffusive transport. If, then, we neglect the convective term in (2.9), $-\theta^{m+1} \partial \theta / \partial z$, we find that (2.14) is reduced to the simpler

$$
\frac{d \Theta}{d \varphi}=-\frac{\Theta^{1-m} \varphi}{m+2}
$$

The solution satisfying $(2.15)$ is

$$
\begin{array}{ll}
m=0, & \boldsymbol{\Theta}=\Theta_{0} \exp \left[-\frac{1}{4} \varphi^{2}\right] \\
m>0, & \boldsymbol{\Theta}=\left[\Theta_{0}^{m}-\frac{m \varphi^{2}}{2(m+2)}\right]^{1 / m} .
\end{array}
$$

We thus retrieve the instantaneous source solutions of Pattle [9] for nonlinear diffusion. These solutions are symmetrical about $\varphi=0$. The approach of our convection-diffusion solutions to these symmetrical shapes as $\boldsymbol{\theta}_{0}$ decreases is observable in Figures 3-6 (see pp. 377-378). Note that the first of (4.25) is the limit of (3.2) for large $\Theta_{0}^{-1}$ as it should be. We observe further that (4.25) implies, for $m>0$ and $\Theta_{0}$ small, that

$$
\varphi_{+}, \varphi_{-} \approx \pm\left[\frac{2(m+2) \Theta_{0}^{m}}{m}\right]^{1 / 2}
$$




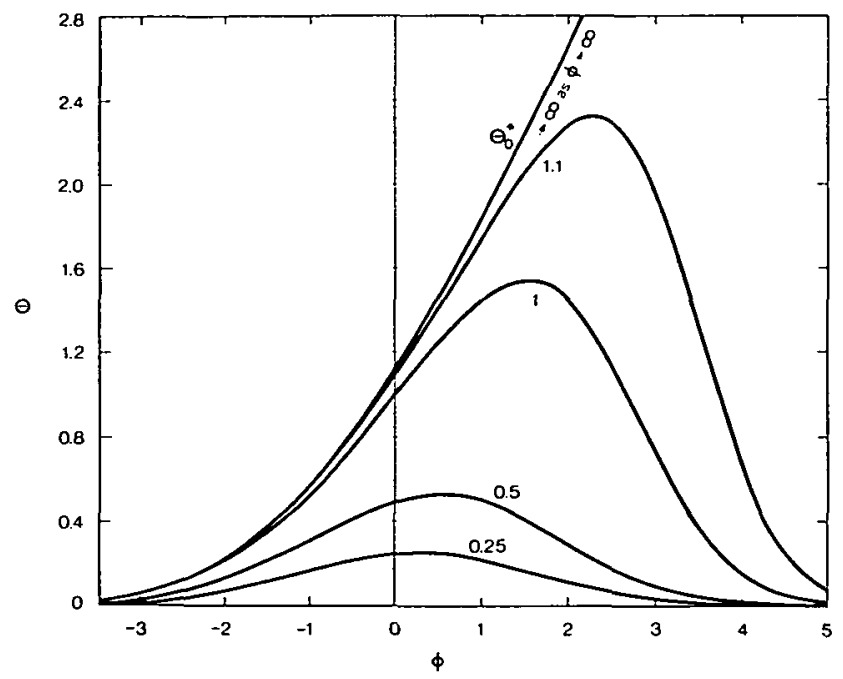

FIGURE 3. Solutions for $m=0$, including the singular case $\theta_{0}=\theta_{0}^{*}=2 \sqrt{\pi} \approx 1.128379$. Numerals on the curves are values of $\Theta_{0}$.

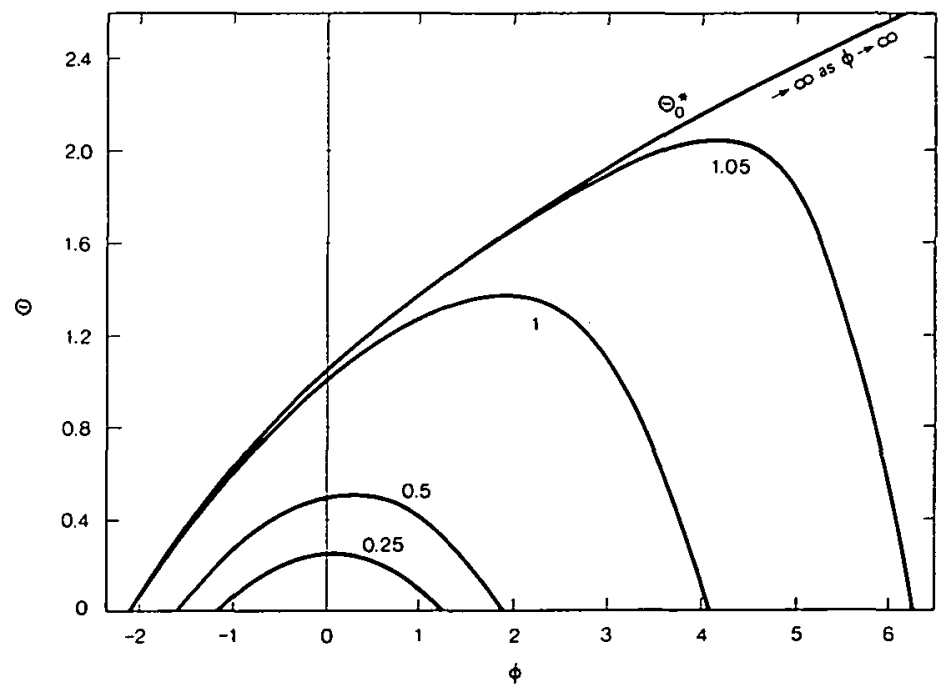

Figure 4. Solutions for $m=1$, including the singular case $\theta_{0}=\theta_{0}^{*}=3^{2 / 3} \Gamma\left(\frac{2}{3}\right) / \Gamma\left(\frac{1}{3}\right) \approx$ 1.051416. Numerals on the curves are values of $\theta_{0}$. 


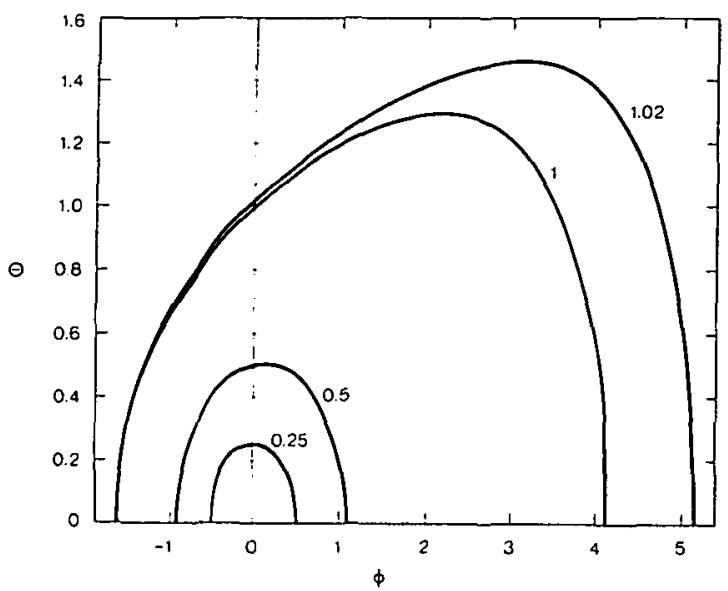

Figure 5. Solutions for $m=2$. Numerals on the curves are values of $\theta_{0}$. Here $\Theta_{0}^{*} \approx$ 1.028 .

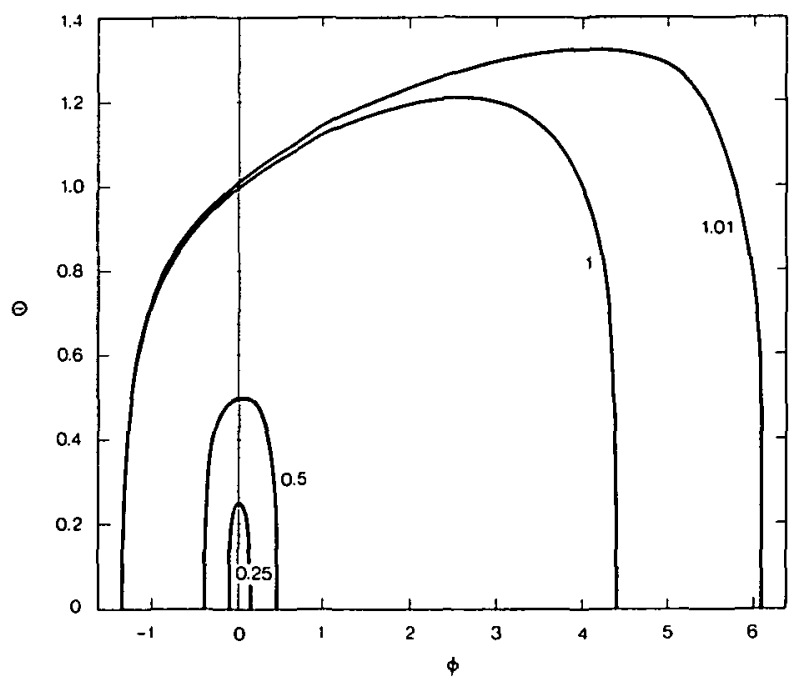

Figure 6. Solutions for $m=4$. Numerals on the curves are values of $\theta_{0}$. Here $\theta_{0}^{*} \approx$ 1.012 . 
and that

$$
Q_{-} \approx Q_{+} \approx \frac{1}{2} Q \approx\left[\frac{2 \pi m}{m+2}\right]^{\frac{1}{2}}\left(\Gamma\left(\frac{m+1}{m}\right) / \Gamma\left(\frac{m+2}{m}\right)\right) \Theta_{0}^{\frac{m+2}{2}} .
$$

Finally, we notice that (4.25) is consistent with (3.24) and (3.25) for the case $m=1$.

\section{Illustrative solutions}

In addition to the analytical results for $m=0$ and 1 , numerical solutions have been developed for $m=1 / 2,2,4$, and 8 . Limited exploratory calculations only were made for $m=1 / 2$ and 8 , with more detailed work done for $m=2$ and 4 . Figures $3,4,5$ and 6 show the solutions obtained for $m=0,1,2$, and 4 respectively.

Solutions for $m=0$, including the singular case $\boldsymbol{\theta}_{0}=\boldsymbol{\theta}_{0}^{*}=2 / \sqrt{ } \pi \approx$ 1.128379. Numerals on the curves are values of $\Theta_{0}$. Solutions for $m=1$, including the singular case $\Theta_{0}=\Theta_{0}^{*}=3^{2 / 3} \Gamma\left(\frac{2}{3}\right) / \Gamma\left(\frac{1}{3}\right) \approx 1.051416$. Numerals on the curves are values of $\Theta_{0}$. Solutions for $m=2$. Numerals on the curves are values of $\theta_{0}$. Here $\theta_{0}^{*} \approx 1.028$. Solutions for $m=4$. Numerals on the curves are values of $\Theta_{0}$. Here $\Theta_{0}^{*} \approx 1.012$.

Numerical solutions were conveniently based on (2.16). They involved backward and forward integration from $u=u_{0}$ at $\varphi=0$, using finite differences. Calculations were for two step sizes, with final estimates using Richardson's $h^{2}$-extrapolation [18]. The larger number of steps ranged from 24 to 112 . Final estimates of $\varphi_{-}$and $\varphi_{+}$were based on analytical approximations in steps at each end of the $\varphi_{-}$range. It is estimated that errors in $\Theta(\varphi)$ do not usually exceed about 1 in 1000 , though in unfavourable circumstances, with $\Theta_{0}$ close to $\Theta_{0}^{*}$ and $\varphi$ large, errors may approach $1 \%$.

\section{Physical remarks}

Two physical remarks are offered on these solutions. Firstly, it will be observed that, as $\theta_{0}$ increases, the asymmetry about $\varphi=0$ of $\theta(\varphi)$ increases. The physical explanation is that increasing $\Theta_{0}$ increases the magnitude of convective effects relative to diffusive effects. Convection operates in the positive $\varphi$ direction and distorts in that direction the distribution of transported material.

These considerations lead on to the second remark, which concerns the finite upper bound, $\boldsymbol{\Theta}_{0}^{*}$, for Problems 1 and 2 . The physical explanation for 
this is, apparently, that when $\Theta_{0} \geq \theta_{0}^{*}$, convection is so strong relative to diffusion that the dynamic equilibrium between the two processes, implied by our similarity solutions, ceases to be possible for these problems. In the absence of the convection term in (2.9), there is no such bound on $\boldsymbol{\theta}_{0}$. Compare Pattle's source solutions [9].

\section{Some related studies}

After this paper was submitted, Professor J. M. Hill kindly furnished me with a preprint of [6]. Among solutions given by Hill and Hill is an instantaneous source similarity solution of (2.4) for (in the present notation) $m=1$. These authors take their dimensionless space variable negative in the direction of the convection, so that their $x$ is equivalent to $-z$ in the present work. Their solution, expressed in Bessel functions, should agree with the present (3.17), but there are important differences. It is stated in [6] that the solution is nonzero only in $z \leq 0$ and is zero in $z>0$. We have, however, physically acceptable nonzero solutions not only in $-\infty \leq z \leq 0$ (Problem 3) but also in $0 \leq z \leq \infty$ (Problem 1) and $-\infty \leq z \leq \infty$ (Problem 2). The confusion in [6] is perhaps the outcome of using $J_{1 / 3}$ and $J_{-1 / 3}$ rather than the more transparent Airy functions. Note that by supposing there is a nonzero solution only in $z \leq 0$, these authors miss an important element of the solutions, the upper bound on $\theta_{0}$ for Problems 1 and 2 .

According to [6], the initial condition is a "delta-function at the origin". A more precise statement is that the initial condition involves the specialised delta-functions defined as follows:

$\begin{array}{lll}\text { Problem 1. } & \lim _{t \rightarrow 0} t^{-\frac{1}{m+2}} \Theta\left(z t^{-\frac{1}{m+2}}\right) / Q_{+}, & 0 \leq z \leq \infty ; \\ \text { Problem 2. } & \lim _{t \rightarrow 0} t^{-\frac{1}{m+2}} \Theta\left(z t^{-\frac{1}{m+2}}\right) / Q, & -\infty \leq z \leq \infty ; \\ \text { Problem 3. } & \lim _{t \rightarrow 0} t^{-\frac{1}{m+2}} \Theta\left(z t^{-\frac{1}{m+2}}\right) / Q_{-}, & -\infty \leq z \leq 0 .\end{array}$

In each case we must take the $\Theta(\varphi)$, and the values of $Q_{+}, Q$, and $Q_{-}$, specific to the particular value of $\boldsymbol{\theta}_{0}$.

A referee has drawn my attention to two other studies of (2.4). The first [8] addresses the special case $n=0$ and employs a similarity scheme quite distinct from that used here. The second [7] involves a similarity scheme similar to but more general than (2.6). Consistent with the present notation, we write it here as

$$
\theta=\Theta(\varphi) t^{\alpha}, \quad \varphi=z t^{\alpha-\beta-1} .
$$

Solutions in $z \geq 0$ are sought which satisfy a time-dependent flow velocity 
condition at $z=0$. We see immediately that similarity requires that the total material in $z \geq 0$ at time $t$ be proportional to $t^{\beta+1}$; that (apart from the zero-flow case with $\beta=-1$, which is Problem 1 of the present work) physically meaningful flows require $\beta>-1$; and that the condition at $z=0$ is a positive flow velocity proportional to $t^{\beta}$. We limit consideration below to the case $\beta>-1$.

Putting (7.2) in (2.4) shows that similarity requires that

$$
n=\frac{m \beta-1}{1+2 \beta} ; \quad \text { i.e. } n-m=-\frac{1+m+m \beta}{1+2 \beta} .
$$

Recalling that we require $m \geq 0, n \geq 0$, to avoid physically unacceptable singularities (often with pathological mathematical consequences) in $D$ and $d K / d \theta$ at $\theta=0$ [cf. (2.1)], we see that the first of (7.3) requires that

$$
\text { either }-1<\beta<-\frac{1}{2} \text { or } \beta \geq \frac{1}{m}>0 \text {. }
$$

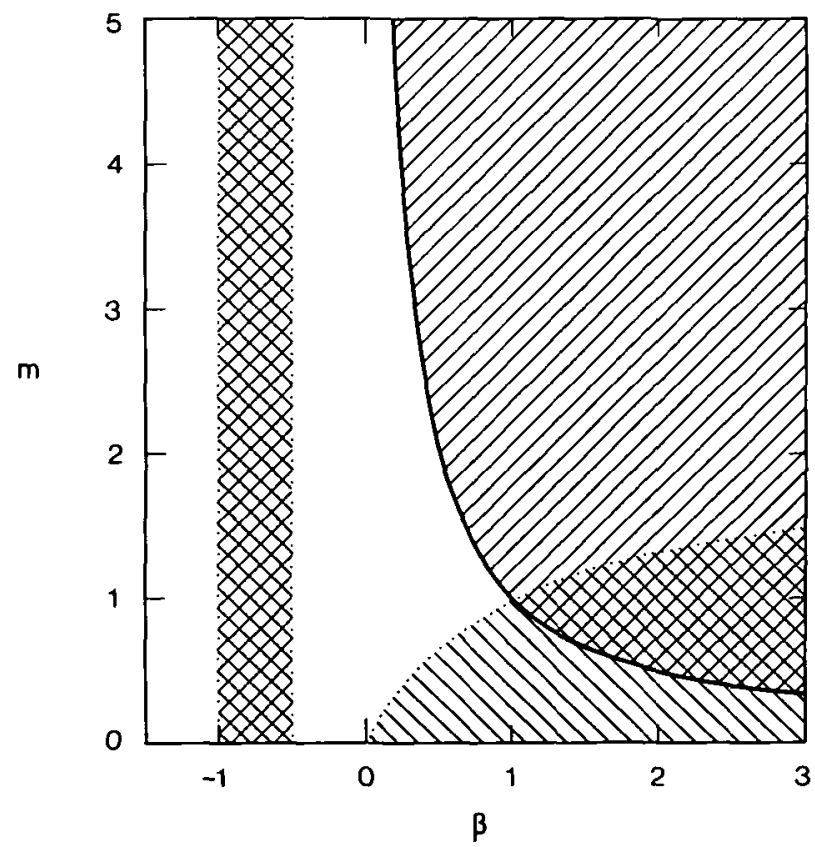

FIGURE 7. Limits on applicability of similarity scheme (7.2) for (2.4). Region with hatching rising to the right satisfies (7.4), i.e. gives $m \geq 0, n \geq 0$, as required. Region with hatching rising to the left satisfies (7.6), i.e. gives $n-m>-1$, as required for soil-water applications. Region with double hatching satisfies both conditions. 
This is a significant limitation on the applicability of (7.2), since most interest attaches to the case $\beta=0$ (constant flow velocity at $z=0$ ) and to cases in the range $-\frac{1}{2} \leq \beta \leq \frac{1}{2}$ giving relatively mild time-dependence of the boundary condition. See Figure 7 .

In the soil-water context the limitation is even more serious. The point at issue is the relation between $m$ and $n$, which determines the form of $d \Psi / d \theta$, the derivation of the moisture potential. Since $D=K d \Psi / d \theta$, $d \Psi / d \theta$ is proportional to $\theta^{m-n-1}$ for $m \geq 0, n \geq 0$. Realistic representation of $d \Psi / d \theta$ requires this quantity to increase as $\theta$ decreases. That is we require, at the least, that

$$
n-m>-1 \text {. }
$$

Applying the second of (7.3) to (7.5) we obtain the requirement that

$$
\text { either }-1<\beta<-\frac{1}{2} \text { or } \beta>-\frac{1}{2}, m<2 \beta /(1+\beta) \text {. }
$$

These inequalities also are indicated on Figure 7. We see that the condition (7.5) gives a further restriction on the ranges of $m$ and $\beta$. Disappointingly, the similarity scheme (7.2) gives few solutions that are physically meaningful and practically relevant. No such restrictions apply to the redistribution problems solved in the present paper.

In [7] the authors arrive at a nonlinear second-order equation in $\Theta(\varphi)$ and give a complicated approximate technique for solving (2.4) in $0 \leq z \leq \infty$.

\section{Acknowledgements}

I am grateful to my colleague Dr. J. H. Knight for helpful discussion, especially of reference [6]. I thank also the Australian Water Resources Advisory Council for the Eminent Researcher Fellowship which supported this work.

\section{References}

[1] H. A. Antosiewicz, “10. Bessel functions of fractional order”, in Handbook of mathematical functions, (eds. M. Abramowitz and I. A. Stegun), (U. S. Govt. Printing Office, Washington, 1964) 437-478.

[2] E. Buckingham, "Studies on the movement of soil moisture", U.S. Dept. Agr. Bur. Soils Bull. 33 (1907).

[3] J. M. Burgers, "A mathematical model illustrating the theory of turbulence", Adv. Applied Mechs. 1 (1948) 171-199.

[4] E. C. Childs, The physical basis of soil water phenomena (Wiley, London, 1969).

[5] W. Gautschi, "7. Error function and Fresnel integrals", in Handbook of mathematical functions, (eds. M. Abramowitz and I. A. Stegun), (U.S. Govt. Printing Office, Washington, 1964) 295-309. 
[6] J. M. Hill and D. M. Hill, "High-order nonlinear evolution equations", IMA J. Appl. Math. 44 (1990) 243-265.

[7] W. L. Hogarth, J.-Y. Parlange, and R. D. Braddock, "First integrals of the infiltration equation: 2. Nonlinear conductivity", Soil Sci. 148 (1989) 165-171.

[8] J.-Y. Parlange and J. F. Fleming, "First integrals of the infiltration equation: 1. Theory", Soil Sci. 137 (1984) 391-394.

[9] R. E. Pattle, "Diffusion from an instantaneous point source with a concentrationdependent coefficient", Q. J. Mech. Appl. Maths. 12 (1959) 407-409.

[10] J. R. Philip, "The theory of infiltration: 3. Moisture profiles and relation to experiment", Soil Sci. 84 (1957) 163-178.

[11] J. R. Philip, "Theory of infiltration", Adv. Hydrosci. 5 (1969) 215-296.

[12] J. R. Philip, "Flow in porous media", Ann. Rev. Fluid Mech. 2 (1970) 177-204.

[13] J. R. Philip, "Flow in porous media", in Theoretical and applied mechanics, (eds. E. Becker and G. K. Mikhailov), Proc. 13th Int. Congr. Theor. Appl. Mech. (Springer, Berlin, 1973) 279-294.

[14] J. R. Philip, "Recent progress in the solution of nonlinear diffusion equations", Soil Sci. 117 (1974) 257-264.

[15] J. R. Philip, “Quasianalytic and analytic approaches to unsaturated flow”, in Flow and transport in the natural environment: advances and applications, (eds. W. L. Steffen and O. T. Denmead), (Springer, Heidelberg, 1988) 30-47.

[16] J. R. Philip, "Infiltration of water into soil", ISI Atlas of Science: Animal and Plant Sciences 1 (1988) 231-235.

[17] L. A. Richards, "Capillary conduction of liquids through porous mediums", Physics 1 (1931) 318-333.

[18] L. F. Richardson, "The deferred approach to the limit, part 1" Phil. Trans. Roy. Soc. London A 226 (1927) 299-349. 